http://jmscr.igmpublication.org/home/ ISSN (e)-2347-176x ISSN (p) 2455-0450 crossref DOI: https://dx.doi.org/10.18535/jmscr/v10i1.17

\title{
Pelizaeus-Merzbacher disease - Classical Form: A rare case report from Katihar Medical College, Katihar, Bihar
}

\author{
Authors \\ Dr Gupta Bhushan Devendrakumar ${ }^{1}$, Dr Ashit Kumar ${ }^{2 *}$, \\ Dr (Professor) Ghazi Sharique Ahmad ${ }^{3}$, Dr Aromita Deb ${ }^{4}$ \\ ${ }^{1}$ Post graduate Student, Pediatrics, KMCH \\ ${ }^{2}$ Assistant Professor, Pediatrics, KMCH \\ ${ }^{3} \mathrm{HOD}$, Pediatrics, KMCH \\ ${ }^{4}$ Post graduate Student, Pediatrics, KMCH \\ *Corresponding Author \\ Dr Ashit Kumar
}

\begin{abstract}
Pelizaeus-Merzbacher disease (PMD) is an X-linked leukodystrophy characterized by developmental delay, nystagmus, hypotonia, spasticity, and variable intellectual deficit. First described clinically in 1885. The causative gene is PLP1. It is classified into three sub-forms based on the age of onset and severity: connatal, transitional, and classic PMD.

Keywords: Pelizaeus-Merzbacher Disease, developmental delayed, hypotonia, intellectual deficit, Head nodding, nystagmus.
\end{abstract}

\section{Introduction:}

Pelizaeus-Merzbacher disorder (PMD) (OMIM312080) is a rare $\mathrm{X}$-linked disorder. The main genetic defect is in PLP gene, which lead to defect in proteolipid expression. It was first described clinically by Pelizaeus in 1885 and later in 1910 same was described by Merzbacher ${ }^{1}$.

It is classified as three types: Connatal, Transitional, and Classic PMD.

The connatal form is the most severe form presenting, since birth, with hypotonia, nystagmus, respiratory distress, and stridor, with subsequent motor and cognitive delay and spastic quadriparesis. The classic form manifests during the first 2 months of life with nystagmus and hypotonia which is progressively replaced by spasticity. Later signs include ataxia, impaired motor development and intellectual deficit. The transitional form is of intermediate severity between the connatal and classic forms. The mildest presentation of PMD (mild developmental and motor delay beginning at 2-3 years old, later associated with spastic paraplegia, ataxia, and/or mild intellectual deficit) is not clearly distinguishable from PLP1 null syndrome, which consists of mild PMD features associated with peripheral neuropathy, and complicated spastic paraplegia 2, a disorder primary characterized by spastic gait in its pure form ${ }^{2}$. 
It is an X-linked disorder due to mutation or dosage alteration of the PLP1 gene (Xq22) that causes hypomyelination of the central nervous system.

Approximately 60-70\% of cases of PMD result from duplications of region of the $\mathrm{X}$ chromosome that contains PLP1. Most individuals with PLP1 duplications present with classic $\mathrm{PMD}^{4}$.

Approximately $15-20 \%$ of mutations in PMD are point mutations or other small mutations that result in base substitutions, insertions, or deletions. Base substitutions usually result in missense mutations, but nonsense mutations and splicing mutations also occur. Splicing mutations are now recognized as quite common and may account for $20 \%$ of point mutations in the PLP1 gene. The most severe form of PelizaeusMerzbacher disease, the so- called conatal form, usually results from missense substitutions ${ }^{4}$.

Mutations that result in spastic paraplegia type 2 are generally missense mutations that do not prevent the processing of DM20, although they may interfere with the processing of PLP itself. These mutations do not appear to cause oligodendrocyte cell death. ${ }^{4}$

\section{Case Presentation}

8-month-old boy, product of non-consanguineous marriage, born after full term pregnancy, Normal vaginal delivery, cried immediately after birth, appropriate for gestational age. Came with complain of 9difficulty in feeding since birth, inability to hold his neck till now, unable to roll over and unable to seat. Vocalization not achieved till now. Seizure occurring after one month of birth.

On examination

Head circumference: $46 \mathrm{~cm}$ (between $-1 \mathrm{SD}$ to -2 SD), Length: $84 \mathrm{~cm}$, Weight: $9.1 \mathrm{~kg}$, MUAC: 15 $\mathrm{cm}$

All anthropometric value within normal range

Feeding difficulty, axial hypotonia and limb hypotonia, Nystagmus present, small penile length with hyperpigmented scrotum. Both testes were present.

On MRI brain features suggestive of bilateral mild diffuse cerebral atrophy with diffuse T2W hyperintensity of entire white matter in bilateral cerebral hemisphere, likely to be due to the hypomyelination white matter.

Genetic study was not done.

Pelizaeus-Merzbacher disease is a rare X-linked inherited disorder characterized by hypomyelination of white matter. Gene involved is PLP1 on long arm of X chromosome (Xq22). In PMD, normal myelination either never occurs or is incomplete.

In 1885, Pelizaeus and later in 1910, Merzbacher described this disorder for the first time ${ }^{3 .}$

PMD has traditionally been divided into four categories: classic, conatal, transitional and adult form (spastic paraplegia) ${ }^{3}$.

The most common form is classic PMD. Our case was also classic PMD. Symptoms in classic PMD usually manifest during infancy, as was in our case.

Our case presented with global developmental delay, hypotonia, feeding difficulty, nystagmus. The symptom starts to appear after few 2 weeks of birth. After than feeding difficulty was gradually increased over time. Hypotonia was more in lower limb. Till now head holding is not achieved.

The most common mutation of PLP1 gene is duplication $(70 \%)$ and the most common clinical phenotype of PMD caused by PLP1 duplication corresponds to classic form of PMD (as was our (ase) $)^{5}$.

MRI brain shows mild diffuse cerebral atrophy with diffuse $\mathrm{T} 2 \mathrm{~W}$ hyperintensity of entire white matter in bilateral cerebral hemisphere most likely due to hypomyelination of white matter.

By above phenotypic description it was classified as classic type of Pelizeaus-Merzbacher disease. Genetic study is required to confirm and classify it. 


\section{JMSCR Vol||10||Issue||01||Page 85-89||January}

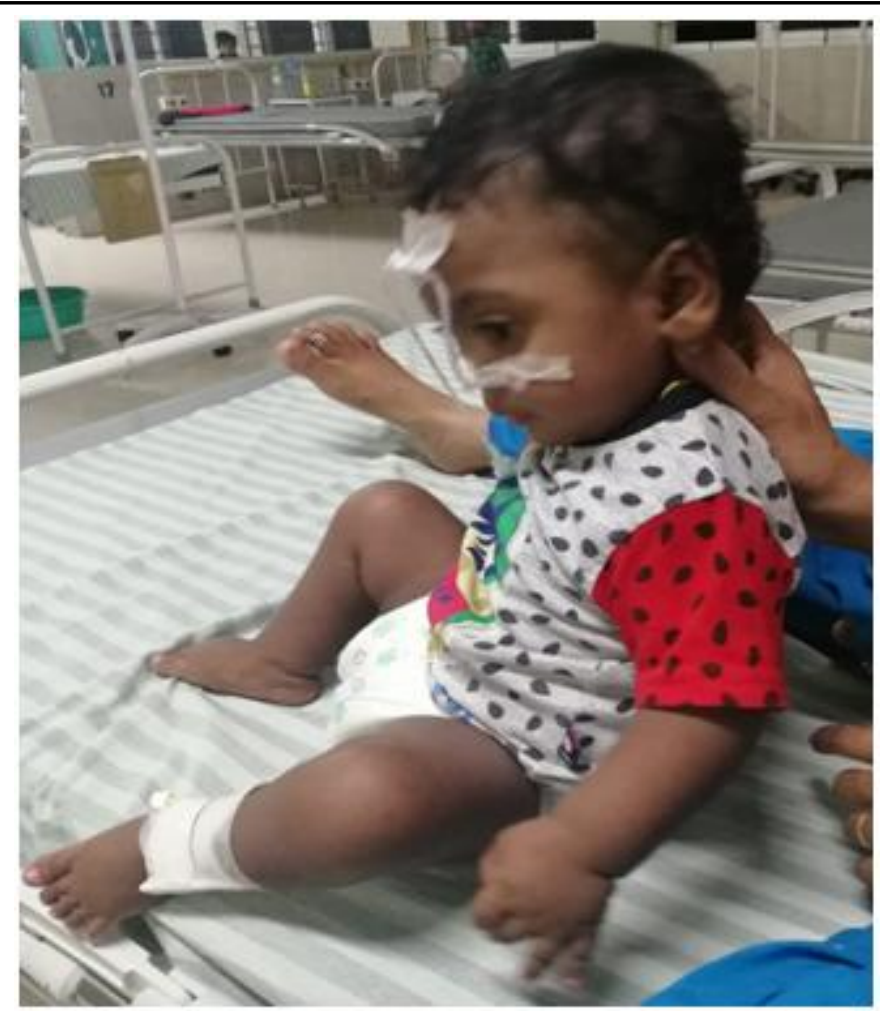

Fig 1: poor head control and axial and limb hypotonia

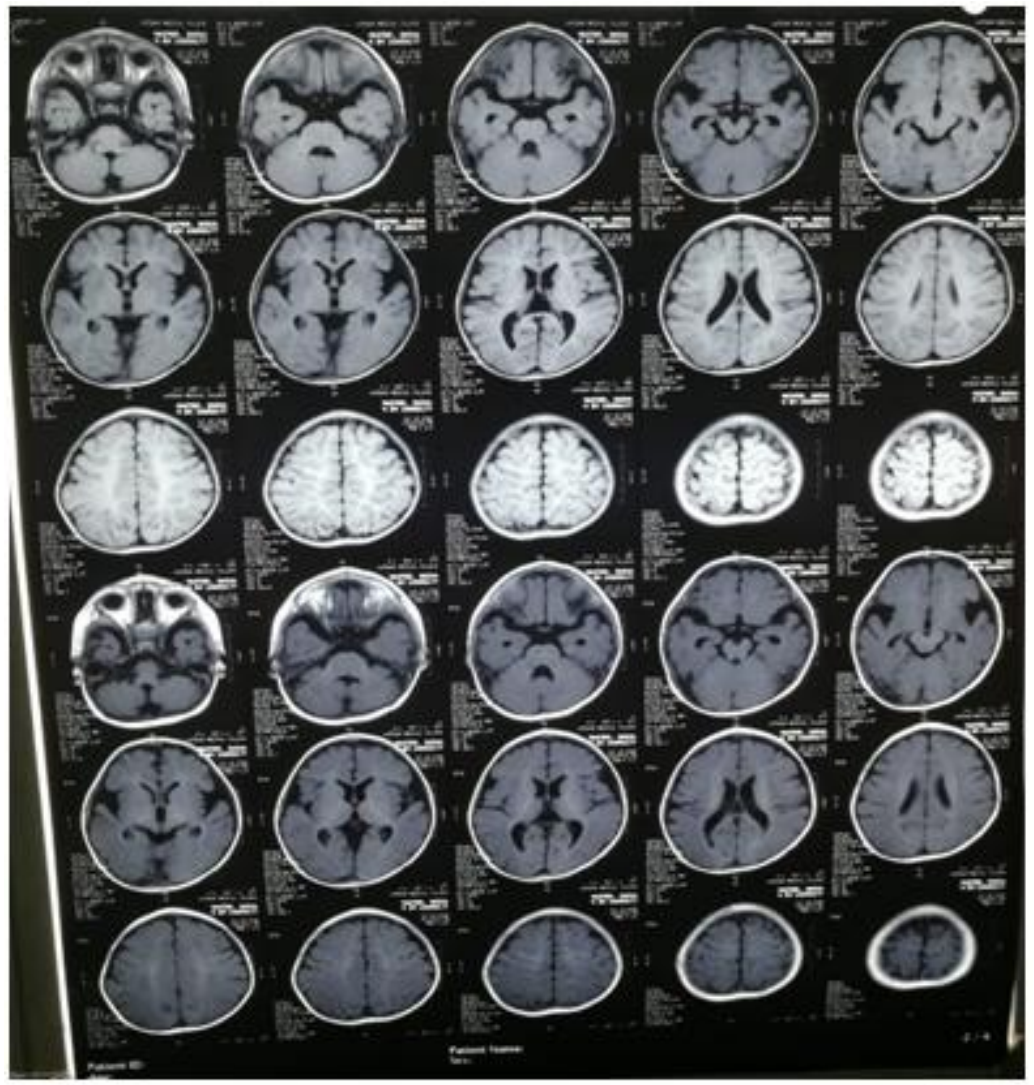

Fig2: MRI brain showing cortical atrophy 


\section{JMSCR Vol||10||Issue||01||Page 85-89||January}
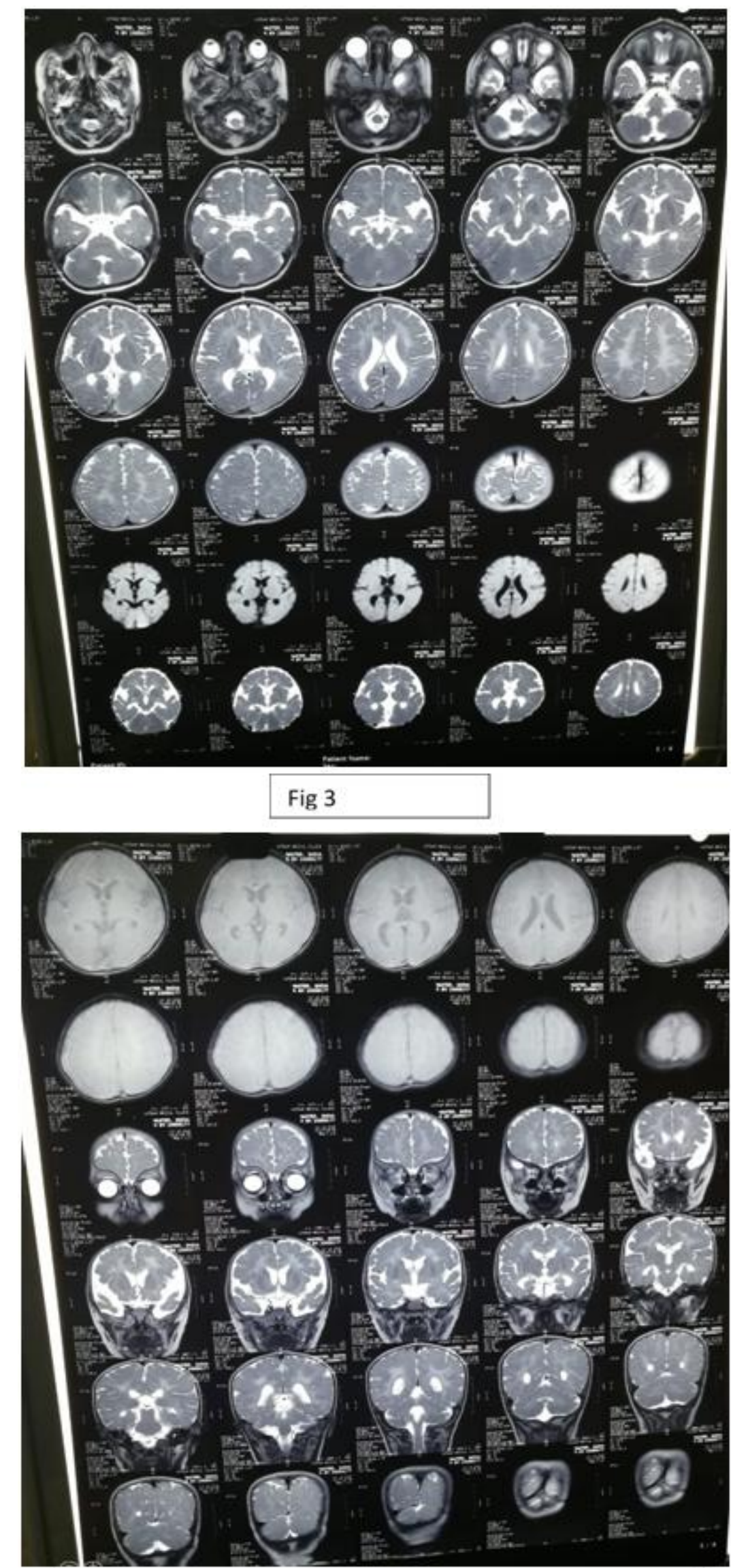

Fig 4:

Fig 3 \&4:MRI brain showing hypomyelination of white matter 


\section{Conclusion}

The possibility of PMD should be considered in male child with global developmental delay, hypotonia, abnormal eye movement in early infancy.

\section{Reference}

1. Ashrafi MR, Mohammad M, Alizadeh H, Nikkhah A; Pelizaeus-Merzbacher Disease: The first genetically approved case report from Iran; Iran J Pediatr, , Sep 2011; vol 21(No 3),Pp:395-398

2. Opharanet: Pelizaeus- Merzbacher disease.

3. Garbern JY. Pelizaeus-Merzbacher disease: genetic and cellular pathogenesis. Cell Mol Life Sci 2007;64(1):50-65.

4. http://emedicine.medscape.com/article/115 3103-overview\#a5

5. Woodward KJ. The molecular and cellular defects underlying Pelizaeus-Merzbacher disease. Expert Rev Mol Med 2008;10:e14. 